\title{
Early experience of coronary artery bypass grafting with a new self-closing clip device
}

\author{
Minoru Ono, MD \\ Randall K. Wolf, MD \\ Dimitrios Angouras, MD \\ E. William Schneeberger, MD
}

\footnotetext{
From the Division of Cardiothoracic Surgery, The Ohio State University Medical Center, Columbus, Ohio.

Received for publication July 12, 2001; revisions requested Aug 28, 2001; revisions received Sept 10, 2001; accepted for publication Sept 12, 2001.
}

Address for reprints: Randall K. Wolf, MD, Division of Cardiothoracic Surgery, The Ohio State University Medical Center, 410 W 10th Ave, Doan Hall-Room N816, Columbus OH 43210 (E-mail: wolf-4@ medctr.osu.edu).

J Thorac Cardiovasc Surg 2002;123:783-7

Copyright (C) 2002 by The American Association for Thoracic Surgery

$0022-5223 / 2002 \$ 35.00+0 \quad \mathbf{1 2 / 1 / 1 2 0 3 3 8}$

doi: $10.1067 / \mathrm{mtc} .2002 .120338$
Objective: We are in the midst of development of several new anastomotic devices for use in coronary artery bypass grafting surgery. This study was designed to examine one of these devices (a new self-closing clip) for left internal thoracic artery-left anterior descending coronary artery anastomosis. Its feasibility and the quality of anastomosis were evaluated.

Methods: Fourteen patients who underwent first-time elective coronary artery bypass surgery were enrolled between July and December 2000. The left internal thoracic artery was anastomosed to the left anterior descending coronary artery in an interrupted manner with Coalescent U-clips (Coalescent Surgical, Inc, Sunnyvale, Calif). Immediate patency was checked with a transit-time flowmeter. Selective angiography was performed 6 months after surgical intervention.

Results: Five patients underwent on-pump coronary bypass grafting, 9 on the beating heart. One patient was excluded from the study intraoperatively because of a poor target site necessitating a 2-cm-long anastomosis. Left internal thoracic artery-left anterior descending artery anastomoses were created with an average of 11.8 clips in 15.9 minutes. Mean graft flow was $45.6 \mathrm{~mL} / \mathrm{min}$. Neither conversion to standard suture technique nor revision of anastomosis was necessary. There tended to be a learning curve in the anastomosis on the beating heart. Postoperative lengths of stay in the intensive care unit and the hospital were 20.7 hours and 3.9 days, respectively. Neither death nor major complication was seen, except for temporary atrial fibrillation in 2 patients. Graft patency at 6 months was $100 \%$ (FitzGibbon grade A).

Conclusion: Left internal thoracic artery-left anterior descending artery anastomoses can be created safely and effectively with new self-closing clips on the beating, as well as the arrested, heart. Midterm patency was shown to be perfect by means of angiography.

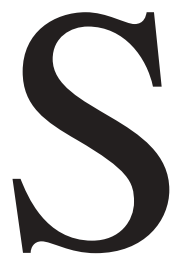

everal attempts to develop an alternative device for coronary artery anastomosis have been reported, including penetrating or nonpenetrating staples, ${ }^{1,2}$ biologic adhesives, ${ }^{3,4}$ and laser welding. 5,6 These alternative anastomotic methods have to date not replaced standard suture anastomosis. The Coalescent U-clip (Coalescent Surgical, Inc, Sunnyvale, Calif) is a new self-closing clip device that has been approved by the Food and Drug Administration. The clip is constructed of a nickel-titanium alloy (nitinol, shape-memory alloy), which can recoil to its original closed state once detached from the delivery mechanism (flexible wire and needle). In the bovine model it was demonstrated that this self-closing clip device performs as intended and that it can be used safely and effectively for the creation of distal coronary anastomoses. ${ }^{7} \mathrm{We}$ designed this clinical study to examine the feasibility of this new self-closing clip for left internal thoracic artery-left anterior descending coronary artery (LITA-LAD) anastomosis and to examine the quality of the anastomosis created. 


\section{TABLE 1. Patient demographics}

\begin{tabular}{lcl}
\hline Age & $64.5 \pm 9.1$ & $44-77$ \\
Female sex & 4 & $30.8 \%$ \\
Diabetes mellitus & 3 & $23.1 \%$ \\
History of CVA & 2 & $15.4 \%$ \\
History of MI & 6 & $46.2 \%$ \\
History of angioplasty & 3 & $23.1 \%$ \\
Body mass index $\left(\mathrm{kg} / \mathrm{m}^{2}\right)$ & $28.6 \pm 2.4$ & $24.1-31.2$ \\
LVEF $(\%)$ & $57.4 \pm 12.0$ & $37-87$ \\
\hline
\end{tabular}

Data are expressed as mean $\pm S D$, number, range, or percentage as shown. CVA, Cerebrovascular accident; $M I$, myocardial infarction; $L V E F$, left ventricular ejection fraction.

\section{Patients and Methods}

Between July and December 2000, 14 patients (4 women) were enrolled in this study at the Ohio State University Medical Center (Table 1). The mean age was $64.5 \pm 9.1$ years, ranging from 44 to 77 years. This study was performed under an investigational device exemption and was approved by the Ohio State University Medical Center Institutional Review Board. Written informed consent was obtained from every patient. All patients required nonemergency, first-time coronary artery bypass grafting (CABG) with or without cardiopulmonary bypass (CPB). Other inclusion criteria were age between 18 and 80 years, body mass index of less than $35 \mathrm{~kg} / \mathrm{m}^{2}$, and left ventricular ejection fraction of $30 \%$ or greater. The exclusion criteria of this study are listed in the Appendix. All parameters examined and shown were collected from 13 patients because one patient was excluded from the study intraoperatively. All data were expressed as means \pm SD.

The patients underwent one of 3 procedures: conventional CABG with $\mathrm{CPB}$, off-pump CABG (OPCAB) through a median sternotomy, or minimally invasive direct CABG (MIDCAB) through a small left anterior thoracotomy.

\section{Conventional CABG}

The LITA was mobilized in a skeletonized fashion. With the aorta clamped, a 4-mm arteriotomy was made on the LAD. Anastomosis was performed in an interrupted fashion with single- or doublearmed U-clips. Additional clips were applied if necessary for bleeding. Other anastomoses were constructed in the usual running fashion. Flow and waveform of each graft were examined with the transit-time flowmeter (Transonic, Ithaca, NY) before and after discontinuation of $\mathrm{CPB}$.

\section{OPCAB}

The LITA was mobilized in the same fashion as for conventional CABG. The heart was presented, and the target coronary artery was exposed. The target vessel was stabilized with the Octopus 3 retractor (Medtronic, Minneapolis, Minn). The LAD was occluded proximally with a tourniquet. The LITA was anastomosed to the LAD with U-clips in the same fashion as mentioned above. Additional clips were used as needed to achieve complete hemostasis. The remainder of the anastomoses were performed in a usual continuous fashion. Flow and waveform of each graft were checked after completion of each anastomosis and before closure of the chest.

\section{MIDCAB}

The LITA was mobilized with an ultrasonic scalpel under videoscopic guidance, as reported elsewhere. ${ }^{8}$ A small left anterior thoracotomy was made in the fourth intercostal space. Stabilization was achieved with a reusable foot device (Genzyme Surgical, Cambridge, Mass). The LAD was occluded proximally with a tourniquet. The LITA was anastomosed to the LAD with U-clips in the same fashion as mentioned above. Additional clips were used as needed to achieve complete hemostasis. Flow and waveform of the LITA graft were checked after completion of anastomosis.

\section{Suture Clip Device}

This nitinol clip device consists of a self-closing clip attached to a needle by means of a flexible member wire with an approximate length of $3 \mathrm{~cm}$ (Figure 1). Two kinds of clips are available: singlearmed and double-armed. The clip is fabricated from nitinol, a shape-memory alloy. It is connected to the flexible wire with a releasing mechanism in between and is kept in the desired $U$ configuration. The needle is pierced into the graft and the coronary artery in an identical fashion as for a conventional suture. Both tissues are approximated, and the open clip is placed at an intended suture point. By compressing a 1-mm-long release mechanism with a needle driver, the clip is deployed, and the releasing clip returns to its original closed loop with an appropriate diameter (Figure 2).

Our current technique using this clip after several attempts is as follows. First, 2 clip devices are placed at the heel. The graft is approximated to the coronary artery, and then clips are deployed. A toe clip is then placed and deployed. The remaining clips are placed on each side of the anastomosis and then deployed.

\section{Follow-up}

Oral aspirin (81 mg daily) was started for all patients on the second postoperative day. Postoperative selective coronary angiography was performed at 6 months after the operation to evaluate the LITA-LAD anastomosis. An experienced cardiologist independently evaluated all angiograms.

\section{Results}

Four conventional CABG, 6 OPCAB, and 4 MIDCAB operations were performed in 14 patients. One patient undergoing an $\mathrm{OPCAB}$ operation was excluded from the study intraoperatively because a long arteriotomy $(2 \mathrm{~cm})$ and on-lay patch-like anastomosis were required for the LAD anastomosis as a result of a heavily calcified artery. A standard continuous technique with 7-0 polypropylene sutures was used for this patient. In the remaining 13 patients, LITALAD anastomosis was completed with U-clips (Table 2). Disease of the LAD at the anastomotic site was severe in 5 patients ( 1 of whom was excluded from the study intraoperatively), moderate in 4 patients, and mild in 5 patients.

The mean number of clips used was $11.8 \pm 1.8$ (range, 915 , including additional clips). No conversion to a standard suture technique was required in these patients. In 6 patients an additional 1 to 3 clips were placed to achieve satisfactory hemostasis. Standard sutures were not used in any patients. 


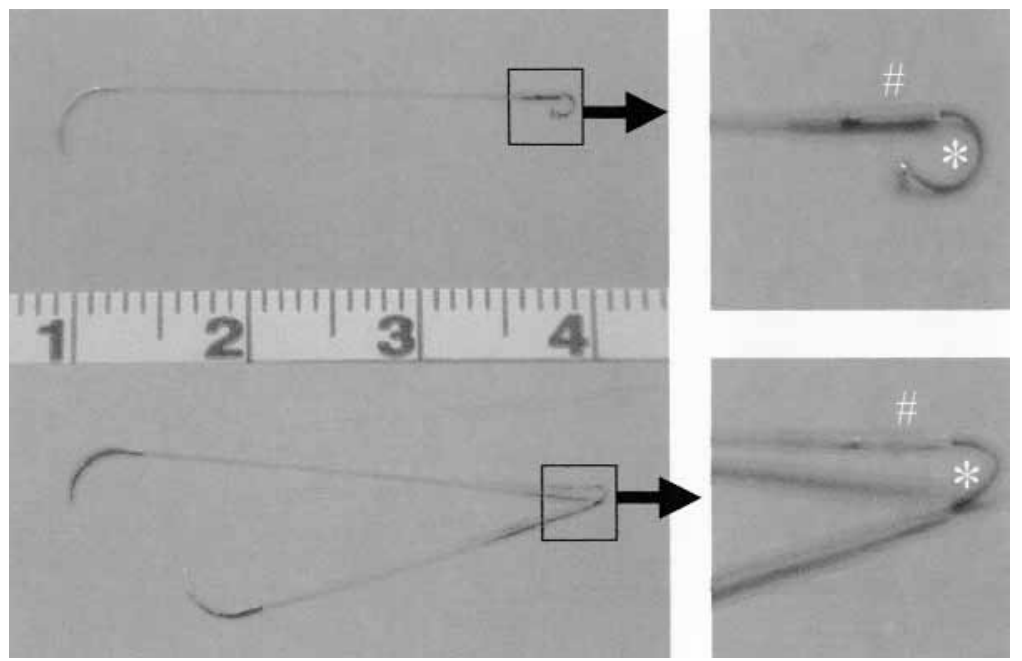

Figure 1. Nitinol self-closing clip device. Top, Single arm; bottom, double arm; \#, releasing mechanism; *clip.
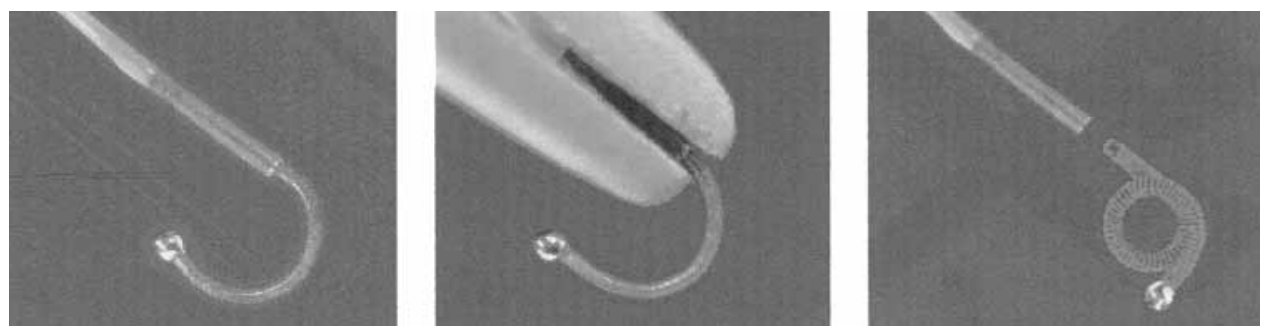

Figure 2. Left, Open clip attached to a releasing mechanism; middle, compression of a releasing mechanism by a needle driver. right, clip that is deployed and returns its original closed loop.

TABLE 2. Results

\begin{tabular}{lcccc}
\hline & & & \multicolumn{1}{c}{ Off-pump } \\
\cline { 3 - 5 } & \multicolumn{1}{c}{ Total } & On-pump & MIDCAB & OPCAB \\
\hline No. of patients & 13 & 4 & 4 & 5 \\
No. of grafts & $2.5(1-5)$ & $3.5(2-5)$ & $17.0 \pm 5.8(11-30)$ \\
Time for anastomosis (min) & $15.9 \pm 5.5(10-30)$ & $12.0 \pm 1.6(10-14)$ & $11.4 \pm 1.7(9-15)$ \\
No. of clips & $11.8 \pm 1.8(9-15)$ & $50.5 \pm 2.1(10-15)$ & $38.2 \pm 18.7(19-100)$ \\
Mean graft flow (mL/min) & $45.6 \pm 24.0(19-100)$ & $2(50 \%)$ & $2(22.2 \%)$ \\
Transfusion requirement & $4(30.8 \%)$ & $2(50 \%)$ & 0 \\
Inotropic support & $2(15.4 \%)$ & $19.3 \pm 2.5(16-22)$ & $21.3 \pm 4.3(13-27)$ \\
Length of ICU stay (h) & $20.7 \pm 3.8(13-27)$ & $5.5 \pm 1.0(4-6)$ & $3.2 \pm 0.7(3-5)$ \\
Length of hospital stay (d) & $3.9 \pm 1.3(3-6)$ &
\end{tabular}

Data are expressed as mean \pm SD. ICU, Intensive care unit.

There was no case of failure to release a clip or insufficient closure of a clip. In 2 beating-heart cases in our early experience, a heel clip was dislodged by inadvertent manipulation of the LITA while turning over its edge. Unnecessary clips were retrieved by using a specially designed removal tool in these patients.

Average time for anastomosis was $15.9 \pm 5.5$ minutes (range, 10-30 minutes). There was a tendency for shorter anastomotic time with the arrested heart compared with that of the beating heart $(12.0 \pm 1.6$ and $17.6 \pm 5.8$ minutes, respectively). Anastomotic time was reduced from $20.3 \pm$ 7.4 minutes in the earlier 4 patients to $15.6 \pm 3.6$ minutes in the latter 5 patients in $9 \mathrm{CABG}$ operations on the beating heart. Flow of the LITA graft was $45.6 \pm 24.0 \mathrm{~mL} / \mathrm{min}(17-$ $100 \mathrm{~mL} / \mathrm{min}$ ), with a diastolic dominant waveform in all cases. Nine $(69.2 \%)$ patients received no perioperative blood 


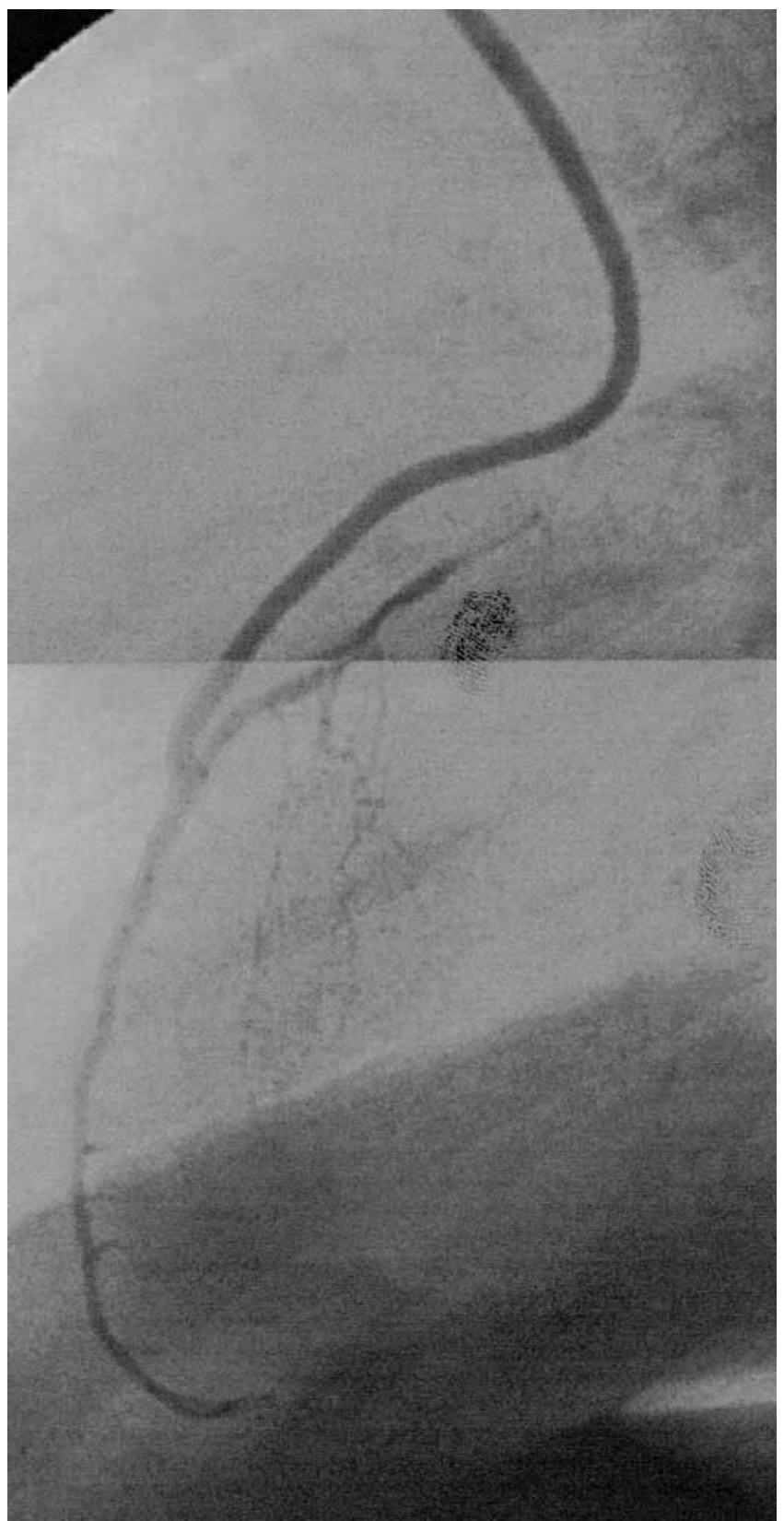

Figure 3. Postoperative angiogram of patient with OPCAB.

transfusions. Inotropic support was necessary for several hours in only 2 patients who underwent on-pump CABG.

Postoperative hospital course was uncomplicated in all but 2 patients, who needed pharmacologic conversion of atrial fibrillation. Length of stay in the intensive care unit and the hospital after the operation were $20.7 \pm 3.8$ hours (range, 13-27 hours) and 3.9 days (range, 3-6 days), respectively. Follow-up angiography was performed in 12 patients. One patient has delayed this follow-up angiogram because of an extenuating family situation. Complete patency of LITA-LAD anastomosis (FitzGibbon grade $\mathrm{A}^{9}$ ) was demonstrated in all patients (Figure 3). All patients are doing well without any ischemic symptoms after a mean follow-up period of $7.0 \pm 1.7$ months (range, 6-10 months).

\section{Discussion}

Several attempts have been reported to develop an alternative device for distal coronary artery anastomosis, including penetrating or nonpenetrating staples, ${ }^{1,2}$ biologic adhesives, ${ }^{4}$ and laser welding. ${ }^{5,6}$ Nataf and colleagues ${ }^{1}$ described the successful clinical application of arcuate-legged, nonpenetrating titanium clips to distal coronary anastomosis in 10 patients. However, symmetric tissue eversion was mandatory for successful application of this nonpenetrating clip, and this clip could not be used in a patient with a heavily calcified coronary artery. Heijmen and coworkers ${ }^{2}$ reported experimental results using the one-shot clip device. The periadventitial tissue of the distal end of the LITA needs to be cleared for loading to the clip cartilage, which may lead to endothelial dysfunction at the anastomotic site and acute vasospasm. The recipient coronary artery needs to be punched out, which may be impractical for diseased vessels.

In the present study satisfactory anastomosis could be achieved, even in patients with a heavily diseased LAD without conversion to a running technique or a repair with a standard suture, except for in one patient in whom an unusually long on-lay patch-like anastomosis was required. The needle of the U-clip has the same strength and quality as that of a standard suture. All clips worked properly as intended in all patients, without any failure to release or inadequate closure. Partial breakdown of the anastomotic heel in our early experience could be avoided easily in later cases by leaving the prior clip device unreleased and obtaining more room for a good visualization for the next device. After completion of the anastomosis, these clips held the tissue around the anastomosis tightly in all cases. The addition of clips to achieve satisfactory anastomosis was performed in the same manner as a standard suture. There tended to be a learning curve for the clip anastomosis on the beating heart.

Hill and colleagues ${ }^{7}$ examined histopathologic changes of the right internal thoracic artery-coronary artery anastomosis created using the same clips on the beating heart in the bovine model. Only one anastomosis showed 10\% stenosis histologically in the animals put to death at 8 weeks among 10 animals ( $\mathrm{n}=8$ at 8 weeks and $\mathrm{n}=2$ at 26 weeks). Microscopic examination of the anastomosis in all animals demonstrated smooth neointimal resurfacing throughout the entire circumference of the inner anastomotic area, proper endothelial cell alignment in the direction of blood flow at the site of exposed clips, and no significant demarcation between the clip and native tissue. ${ }^{7}$ In the present study all patients were free from ischemic symptoms at $7.0 \pm 1.7$ months after the operation, and perfect patency (FitzGibbon 
grade A) was demonstrated in all 12 patients at 6 months. On the basis of these experimental and clinical results, nitinol clips exposed to the bloodstream do not seem to adversely affect the quality and the patency of the anastomosis.

This clip device can eliminate suture management and knot tying, which are inherent to a standard suture. As previously described, an interrupted suture technique avoids the chance of the purse-string effect. ${ }^{10}$ This clip may also be preferable for heavily diseased vessels because a fresh needle is used for every stitch. These advantages can be best used in totally endoscopic computer-enhanced CABG: Suture management is difficult and time consuming in totally endoscopic computer-enhanced CABG. ${ }^{11}$ Knot tying is still challenging without appropriate tactile feedback from the end effector. We have been impressed with the ease of use of this self-closing clip in our beating-heart totally endoscopic CABG experiments. Elimination of manipulations inherent to running sutures may have a positive effect on the advancement of this evolving technology.

In conclusion, the feasibility of a new self-closing clip device for LITA-LAD anastomosis both on the beating and on the arrested heart was demonstrated in this clinical study. This clip did not adversely affect the graft patency and quality at the midterm period (6 months). Further study with a larger number of patients and longer follow-up period is warranted.

\section{References}

1. Nataf P, Kirsch W, Hill AC, et al. Nonpenetrating clips for coronary anastomosis. Ann Thorac Surg. 1997;63:S135-7.

2. Heijmen RH, Hinchliffe P, Borst C, et al. A novel one-shot anastomotic stapler prototype for coronary bypass grafting on the beating heart: feasibility in the pig. J Thorac Cardiovasc Surg. 1999;117:117-25.

3. Bowen CV, Leach DH, Crosby NL, Reynolds R. Microvascular anastomoses. A comparative study of fibrinogen adhesive and interrupted suture technique. Plast Reconstr Surg. 1996;97:792-800.

4. Gundry SR, Black KB, Izutani H. Sutureless coronary artery bypass with biologic glue anastomosis: preliminary in vivo and in vitro results. J Thorac Cardiovasc Surg. 2000;120:473-7.

5. Tulleken CA, Verdaasdonk RM, Mansvelt Beck HJ. Nonocclusive excimer laser-assisted end-to-side anastomosis. Ann Thorac Surg. 1997;63:138-42.

6. Phillips AB, Ginsburg BY, Shin SJ, Soslow R, Ko W, Poppas DP. Laser welding for vascular anastomosis using albumin solder: an approach for MID-CAB. Lasers Surg Med. 1999;24:264-8.

7. Hill AC, Maroney TP, Virmani R. Facilitated coronary anastomosis using a nitinol U-clip device: bovine model. $J$ Thorac Cardiovasc Surg. 2001;121:859-70.

8. Ohtsuka T, Wolf RK, Hiratzka LF, Wurnig P, Flege JB Jr. Thoracoscopic internal mammary artery harvest for MIDCAB using the harmonic scalpel. Ann Thorac Surg. 1997;63:S107-9.

9. FitzGibbon GE, Burton JR, Leach AJ. Coronary bypass graft fate. Angiographic grading of 1400 consecutive grafts early after operation and of 1132 after one year. Circulation. 1978;57:1070-4.

10. Lytle BW. Anastomotic techniques. Operative Techniques Thorac Cardiovasc Surg. 2000;5:222-30.

11. Falk V, Diegeler A, Walther T, et al. Total endoscopic computer enhanced coronary artery bypass grafting. Eur J Cardiothorac Surg. 2000; 17:38-45.

\section{APPENDIX. Exclusion criteria}

\section{Preoperative exclusion criteria}

Severe cerebrovascular disease, including history of stroke within 1 month

History of renal insufficiency (serum creatinine level $>2 \mathrm{mg} / \mathrm{dL}$ )

Active gastrointestinal bleeding

Active infection or fever $\left(>38.3^{\circ} \mathrm{C}\right)$, which may be caused by infection

Short life expectancy (<2 years) as a result of cancer or pulmonary, hepatic, or renal disease

Significant anemia (hemoglobin level $<8.0 \mathrm{~g}$ )

Severe, uncontrolled systemic hypertension (systolic pressure $>240 \mathrm{~mm} \mathrm{Hg}$ within 1 month)

Severe electrolyte imbalance

Documented anaphylaxis during previous exposure to angiographic contrast media

Congestive heart failure

Severe systemic disease

History of pericarditis, median sternotomy, or chest irradiation

Uncontrolled diabetes ( $\geq 2$ glucose levels $>350 \mathrm{mg} / \mathrm{dL}$ within 7 days)

Bleeding diathesis

Vasculitis

History of intravenous drug use within prior year

Participating in another investigational protocol

Unwilling or unable to comply with any protocol requirements

Intraoperative exclusion criteria

Inadequate estimated LITA flow

Small LAD size at anastomotic site $(<1 \mathrm{~mm})$

Inadequate or damaged LITA

Severe intramyocardial LAD (off-pump cases)

Other presenting complications prohibiting use of either LITA or LAD

Unexpected findings of any intraoperative exclusion

Unexpected findings creating an unreasonable intraoperative risk or an increased probability of postoperative complications 